Table 2. ROC analysis of the performance of anti-CCP using various ultrasound erosion score criteria

\begin{tabular}{lc}
\hline Ultrasound erosion score criterion & Area under the ROC Curve (AUC) $(95 \% \mathrm{Cl})$ \\
\hline$>2.25{\left.\text { ( } 25^{\text {th }} \text { percentile }\right)}^{\text {th }}$ & $0.57(0.26,0.87)$ \\
$>4.5{\left.\text { (median } \text { or } 50^{\text {th }} \text { percentile }\right)}^{\text {th }}$ percentile $)$ & $0.68(0.40,0.95)$ \\
& $0.72(0.26,0.97)^{1}$ \\
\hline
\end{tabular}

${ }^{1}$ Corresponding Threshold=95.2, Specificity $=53.8 \%$, Sensitivity $=83.3 \%$, Accuracy $=63.2 \%$, Negative

Predictive Value $=87.5 \%$, Positive Predictive Value $=45.5 \%$.

Figure 1. Scatter plots for RF and anti-CCP levels with ultrasound erosion scores
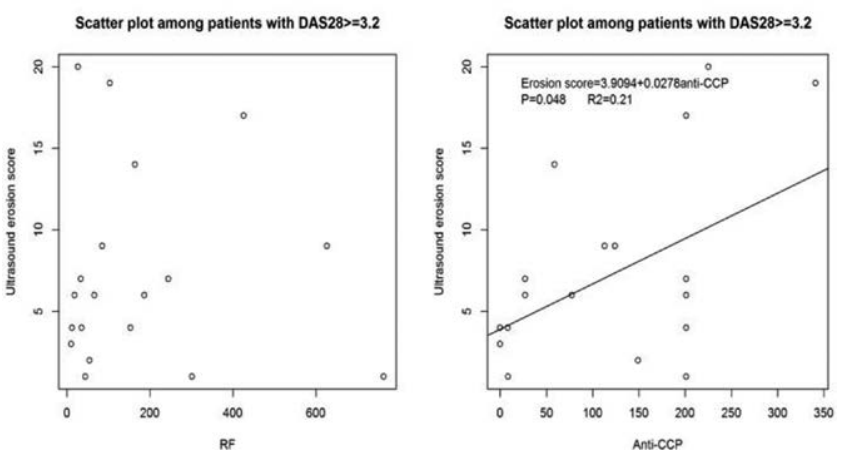

Conclusion: The prognostic significance of anti-CCP and RF appears to differ in RA. Specifically, among patients with at least moderate disease activity (DAS28 $\geq 3.2$ ), anti-CCP - but not RF - is associated with joint damage, being moderately correlated with US detected erosions.

References: Nil:

Disclosure of Interests: None declared

DOI: 10.1136/annrheumdis-2020-eular.171

\section{AB1134 $\quad$ ULTRASOUND FINDINGS IN HAND JOINTS INVOLVEMENT: A COMPARATIVE STUDY BETWEEN PSORIATIC AND RHEUMATOID ARTHRITIS}

A. R. Ahmed ${ }^{1}$, S. Tharwat ${ }^{1}$, A. M. Abd el-Khalek ${ }^{2}$, E. E. Eltoraby ${ }^{1}$ on behalf of No. ${ }^{1}$ Faculty of Medicine, Mansoura University, Rheumatology and Immunology Unit, Internal Medicine department, Mansoura, Egypt; ${ }^{2}$ Faculty of Medicine, Mansoura University, Diagnostic Radiology, Mansoura, Egypt

Background: Psoriasis is a common skin disease that is associated with multiple conditions. The most prevalent coexisting condition is psoriatic arthritis (PsA) which develops in up to $30 \%$ of patients with psoriasis and characterized by diverse clinical features often resulting in delayed diagnosis and treatment. [1] Objectives: The aim of this study was to investigate the potential of ultrasound (US) in the differential diagnosis between rheumatoid arthritis (RA) and PSA at the level of small joints of the hand and wrist.

Methods: Fifteen patients with PsA of the hands and wrists and 20 age and sex-matched RA patients were examined with musculoskeletal US. Radiocarpal, midcarpal, distal radioulnar, metacarpophalangeal (MCP), proximal interphalangeal (PIP) and distal interphalangeal (DIP) joints and flexor and extensor tendons (in wrist and hand) were examined bilaterally. Synovitis, erosions and tenosynovitis were scored according to semiquantitative method.[2]

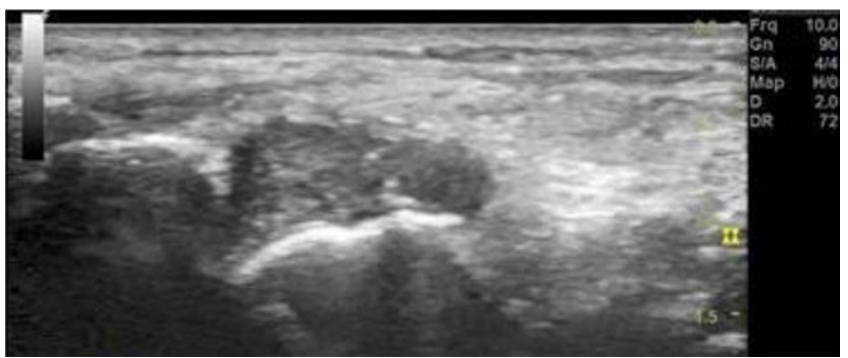

Figure 1 Sixty year old male patient with RA for 5 years,presented with pain and swelling at the wrist.Gray scale ultrasound sagittal view shows sever synovial proliferation at the radiocarpal joint
Results: Among 510 and 680 joints examined in PsA and RA respectively, certain US features such as synovitis and erosions at the DIP were exclusively detected in PsA $(p<0.001)$. Synovitis was frequently present at the radioulnar joints in RA in comparison to PsA patients ( $52.5 \%$ vs $26.7 \%$ respectively, $p=0.029)$. Joint effusion was frequently detected at radiocarpal and midcarpal joints in RA in comparison to PsA ( $p=0.047,0.039$ respectively). Effusion at the $3^{\text {rd }}$ PIP joints was more significantly present in PSA than RA ( $p=0.037)$, while erosions were significantly detected at radiocarpal joints in RA in comparison to PsA patients (45\%vs $20 \%$ respectively,p=0.029). Tenosynovitis was significantly detected at the extensor tendons in RA and at the flexor tendons in PsA patients ( $p=0.021,0.022$ respectively).

Conclusion: There are significant differences in the US findings of the hand and wrist that can help to distinguish between RA and PsA

\section{References:}

[1] Ritchlin, C.T., R.A. Colbert, and D.D. Gladman, Psoriatic arthritis. New England Journal of Medicine, 2017. 376(10): p. 957-970.

[2] Szkudlarek, M., et al., Interobserver agreement in ultrasonography of the finger and toe joints in rheumatoid arthritis. Arthritis \& Rheumatism: Official Journal of the American College of Rheumatology, 2003. 48(4): p. 955-962.

Acknowledgments: Not applicable

Disclosure of Interests: None declared

DOI: 10.1136/annrheumdis-2020-eular.4810

\section{AB1135 ECHO-ANATOMY OF THE PROXIMAL TIBIOFIBULAR JOINT}

M. C. Trojani ${ }^{1}$, B. Le Goff ${ }^{2} .{ }^{1}$ Hospital Pasteur, Nice, France; ${ }^{2}$ Nantes University Hospital Hotel-Dieu, Nantes, France

Background: The proximal tibiofibular joint (PFTJ) should be considered in the differential diagnosis of a patient presenting with complaints in the lateral aspect of the knee However, this joint is often forgotten, yet involved in many degenerative and inflammatory pathological processes. MRI remains the imaging of choice to study the PFTJ. Ultrasound could also be useful in clinical practice to study the joint and its environment. To our knowledge, there is no systematic descriptive echo-anatomical study of PFTJ that would allow to standardize the ultrasound scanning of this joint. Objectives: The objective of our study was to describe standardized ultrasonographic scans of the PTF joint and its environment starting from an anatomica study of the joint and then confirming the visibility of the different structures on a series of healthy volunteers.

Methods: We first conducted an anatomical study of the PTFJ on 3 cadavers. The different part of the joint (capsule, cartilage, ligaments) and the environment (nerves, muscles, vessels) were studied allowing an exact correlation between US images and the structures. This step led us to choose 3 scans useful for the study of the different part of the joint in clinical practice (figure 1): an anterior transverse oblique, a strict coronal, and a posterior transverse oblique. Subsequently, a TFPJ ultrasound was performed on 20 healthy volunteer patients to evaluate the feasibility and the visibility of the different structures seen on the dissection part.
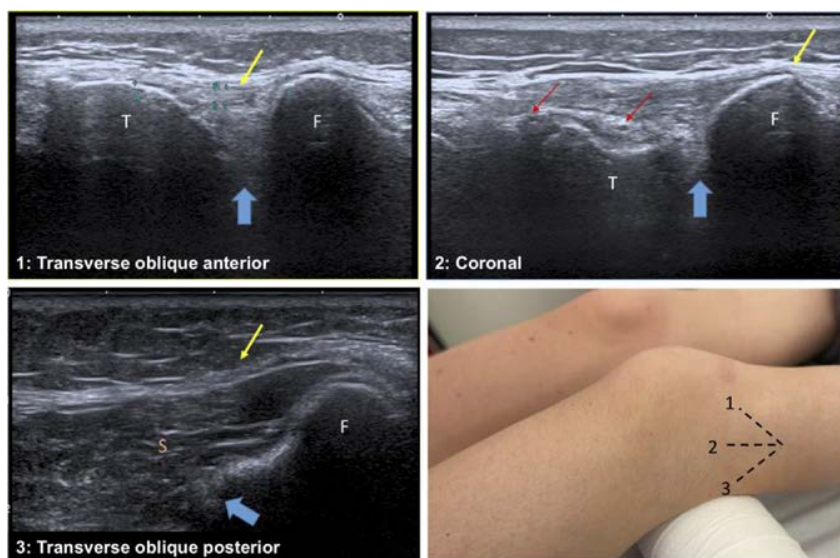

Figure 1.

Results: The different structures seen on the anterior transverse oblique scan were the anterior joint space, cartilage and anterior proximal tibiofibular ligament. The coronal approach led us to the visualization of the joint space, the collatera lateral ligament, the inferolateral genicular and posterior tibial recurrent artery, the meniscus and more posteriorly the ligaments of the posterolateral corner (popliteofibular, arcuate and fabellofibular). Finally, the posterior transverse oblique allowed us to study the posterior ligaments and joint recess under the 